\title{
Ventricular mass index using magnetic resonance imaging accurately estimates pulmonary artery pressure
}

\author{
T.S. Saba*, J. Foster", M. Cockburn ${ }^{\#}$, M. Cowan", A.J. Peacock*
}

Ventricular mass index using magnetic resonance imaging accurately estimates pulmonary artery pressure. T.S. Saba, J. Foster, M. Cockburn, M. Cowan, A.J. Peacock. C ERS Journals Ltd 2002.

ABSTRACT: Magnetic resonance imaging (MRI) can provide accurate anatomical measurements of the cardiac ventricles. This study investigated whether a calculated ventricular mass index (VMI) would provide an accurate means of estimating pulmonary artery pressure noninvasively, and compared the results with conventional Doppler echocardiography and invasive measurement.

A total of 26 subjects referred for investigation of pulmonary hypertension were studied by MRI and echocardiography within 2 weeks of cardiac catheterisation. The correlations for mean pulmonary artery pressure were as follows: VMI (ratio of right ventricular mass over left ventricular mass) $\mathrm{r}=0.81$; pulmonary artery systolic pressure (echocardiography) $r=0.77$. The confidence intervals for the VMI were narrower than for echocardiography. Sensitivity and specificity for pulmonary hypertension were 84 and $71 \%$ respectively for the VMI compared with 89 and $57 \%$ for echocardiography.

The calculated ventricular mass index provides an accurate and practical means of estimating pulmonary artery pressure noninvasively in pulmonary hypertension and may provide a more accurate estimate than Doppler echocardiography. This may be because it reflects the right ventricular response to sustained pulmonary hypertension over a long period and is not influenced by short-term physiological variables affecting echocardiography, such as heart rate, posture, hydration status and oxygen supplementation.

Eur Respir J 2002; 20: 1519-1524.
*Scottish Pulmonary Vascular Unit and ${ }^{\#}$ Dept of Radiology Western Infirmary, Glasgow, Scotland, UK.

Correspondence: A.J. Peacock

Scottish Pulmonary Vascular Unit

Level 8 Laboratory

Western Infirmary

Dumbarton Road

Glasgow

G11 6NT

Scotland

UK

Fax: 441412116334

E-mail: apeacock@udcf.gla.ac.uk

Keywords: Calculated ventricular mass index

Doppler echocardiography

magnetic resonance imaging

noninvasive

pulmonary arterial hypertension

Received: February 202002

Accepted after revision: June 252002
Pulmonary arterial hypertension (PAHT) is a rare disorder characterised by high pulmonary vascular resistance. Prognosis is related to mean pulmonary artery pressure (MPAP) measured at right heart catheterisation (RHC) [1], and repeated measurements are often necessary to assess disease progression and the response to treatment. The most widely used noninvasive technique, Doppler echocardiography (ECHO) [2] is safe and widely available, but has several limitations. Firstly, it cannot measure MPAP and only provides an estimate of pulmonary artery systolic pressure (PASP). Secondly, it depends upon the presence of detectable tricuspid regurgitation and has a significant failure rate in some patient groups [3]. Finally, measurements are somewhat operatordependent, and influenced by physiological variables such as heart rate, hydration status and posture, limitations that also affect the accepted gold standard method of RHC [4].

Magnetic resonance imaging (MRI) is an attractive modality for studying the complex geometry of the right ventricle and pulmonary vasculature since no assumptions need to be made about the shape or location of the structure being studied. It provides three-dimensional anatomical measurements of right ventricular morphology that are unaffected by physiological variables and more likely to be reproducible than dynamic, planar measurements made at ECHO. Furthermore, these anatomical variables assess the right ventricular response to chronic pulmonary vascular disease and may provide a more clinically relevant assessment of disease severity.

MRI has been extensively used to image the left ventricle but there have been relatively few studies of right ventricular function and morphology. It has been successfully used to quantify ventricular volumes and estimate ventricular mass in both normal and PAHT subjects and normal ranges have been established [5-8]. Noninvasive assessments of blood flow and distensibility in the pulmonary arteries can be made [9-11], as well as estimates of stroke volume and cardiac output [10]. Changes in right ventricular mass (RVM), function and pulmonary artery blood flow have been observed following lung transplantation [12-15] and MRI has been shown to be far more accurate than ECHO in monitoring changes in left ventricular mass (LVM) [16].

Several investigators have attempted to use MRI as a noninvasive means of estimating MPAP but none have reported any advantages over ECHO. Right ventricular end-diastolic wall thickness has been shown to correlate well with MPAP in primary 
Table 1.-Patient demographics and diagnoses

\begin{tabular}{|c|c|c|c|c|c|c|c|}
\hline Patient & Sex & Age yrs & Diagnosis & Other & $\begin{array}{l}\text { MPAP } \\
\mathrm{mmHg}^{\#}\end{array}$ & $\begin{array}{l}\text { Cardiac output } \\
\mathrm{L} \cdot \mathrm{min}^{-1}\end{array}$ & $\begin{array}{c}\text { ECHO PASP } \\
\mathrm{mmHg}\end{array}$ \\
\hline 1 & $\mathrm{~F}$ & 40 & $\mathrm{PPH}$ & SHT & 52 & 3.4 & 58 \\
\hline 2 & $\mathrm{M}$ & 42 & PPH & & 63 & 3.3 & 144 \\
\hline 3 & M & 51 & CTD & & 40 & 3.5 & 64 \\
\hline 4 & $\mathrm{~F}$ & 53 & No PAHT & & 16 & 5.7 & 49 \\
\hline 5 & $\mathrm{~F}$ & 56 & PPH & & 61 & 3.7 & 112 \\
\hline 6 & $\mathrm{~F}$ & 32 & No PAHT & & 19 & 5.1 & $<25$ \\
\hline 7 & $\mathrm{~F}$ & 50 & СТЕРНT & & 27 & 3.9 & 41 \\
\hline 8 & $\mathrm{~F}$ & 38 & CHD & & 78 & 5.5 & \\
\hline 9 & M & 29 & PP & & 36 & 6.7 & 52 \\
\hline 10 & $\mathrm{~F}$ & 44 & CHD & & 66 & 4.7 & 121 \\
\hline 11 & $\mathrm{~F}$ & 54 & No PAHT & & 15 & 6.0 & 36 \\
\hline 12 & $\mathrm{~F}$ & 42 & No PAHT & & 19 & 4.1 & 16 \\
\hline 13 & $\mathrm{~F}$ & 58 & PPH & SHT & 31 & 4.6 & 25 \\
\hline 14 & $\mathrm{~F}$ & 64 & No PAHT & IHD & 16 & 4.4 & 27 \\
\hline 15 & $\mathrm{~F}$ & 33 & CHD & & 78 & 6.2 & 74 \\
\hline 16 & $\mathrm{M}$ & 58 & CTD & LTOT, IHD & 44 & 2.7 & 88 \\
\hline 17 & $\mathrm{~F}$ & 61 & CTD & LTOT & 34 & 2.8 & 100 \\
\hline 18 & $\mathrm{M}$ & 62 & PPH & SHT & 53 & 3.6 & 104 \\
\hline 19 & M & 51 & COPD & & 29 & 3.7 & 52 \\
\hline 20 & $\mathrm{M}$ & 60 & COPD & LTOT, IHD & 39 & 4.8 & 50 \\
\hline 21 & M & 66 & PPH & LTOT & 40 & 2.6 & 74 \\
\hline 22 & $\mathrm{~F}$ & 31 & PP & & 41 & 6.3 & \\
\hline 23 & $\mathrm{~F}$ & 48 & No PAHT & & 16 & 3.4 & 23 \\
\hline 24 & $\mathrm{~F}$ & 68 & CTD & & 35 & 5.4 & 58 \\
\hline 25 & $\mathrm{~F}$ & 60 & No PAHT & & 16 & 6.8 & 38 \\
\hline 26 & $\mathrm{~F}$ & 57 & HHT & & 53 & 6.4 & 52 \\
\hline
\end{tabular}

MPAP: mean pulmonary artery pressure; ECHO: echocardiography; PASP: pulmonary artery systolic pressure; M: male; F: female; PPH: primary pulmonary hypertension; CTD: connective tissue disease; PAHT: pulmonary arterial hypertension; CTEPHT: chronic thromboemboembolism; CHD: congenital heart disease; PP: portopulmonary disease; COPD: chronic obstructive pulmonary disease; HHT: hereditary haemorrhagic telangiectasia; SHT: systemic hypertension; IHD: ischaemic heart disease; LTOT: long-term oxygen therapy. ${ }^{\#}$ : right heart catheterisation.

pulmonary hypertension $(\mathrm{PPH})$ and some causes of secondary pulmonary hypertension [17-19]. A linear relationship between RVM and MPAP has been described for PPH but not for other forms of PAHT [8]. The ratio of main pulmonary artery diameter over descending aortic diameter has also been shown to correlate with MPAP in PPH [20].

In this study, the authors investigated whether a calculated ventricular mass index (VMI) measured with MRI, and not previously described in PAHT, can provide an accurate means of estimating MPAP noninvasively in subjects with both $\mathrm{PPH}$ and a wide range of types of secondary PAHT undergoing routine invasive RHC. The accuracy of these estimates were then compared with those made at ECHO.

\section{Materials and methods}

\section{Study subjects}

This study was approved by West Glasgow Hospitals NHS Trust Ethics Committee. All patients referred to the Scottish Pulmonary Vascular Unit for investigation of suspected PAHT September 1999March 2001 were considered for enrolment. Patient selection depended upon compliance with MRI safety criteria, the ability to lie flat and breath-holding ability. Twenty-six subjects (table 1) were enrolled after informed consent was obtained. All had normal left ventricular function and morphology at ECHO and normal pulmonary artery occlusion pressures.

PAHT was confirmed in 19 subjects (table 1), of whom six had PPH, four connective tissue disease, three congenital heart disease, two chronic obstructive pulmonary disease, two portopulmonary disease, one chronic thromboemboembolism and one hereditary haemorrhagic telangiectasia. The remaining seven had normal pulmonary haemodynamics at RHC, and had been referred because of evidence of PAHT at ECHO (subjects 4, 6, 11, 12 and 25) or cardiopulmonary exercise testing (subjects 14 and 23).

\section{Study design}

MRI and ECHO were performed within 2 days of RHC where possible, and always within 2 weeks.

\section{Methods}

Right heart catheterisation. This was performed according to standard protocols using a balloontipped, thermodilution cardiac catheter (Swan Ganz, 7-F, Baxter Healthcare, Irvine, CA, USA). 
Measurements were made on supplementary oxygen for those on long-term oxygen therapy (LTOT) (subjects 16, 17, 20 and21). PAHT was defined as MPAP of $\geqslant 25 \mathrm{mmHg}$.

Doppler echocardiography. PASP was estimated from tricuspid regurgitation in the conventional way (Acuson Sequoia c256, Mountain View, CA, USA) [2]. Measurements were made on supplementary oxygen for those on LTOT. PAHT was defined as PASP of $\geqslant 35 \mathrm{mmHg}$.

Magnetic resonance image acquisition. MRI scans were performed using a standard scanner (Impact Expert $1.0 \mathrm{~T}$, Siemens Medical Engineering, Erlangen, Germany). Imaging sequences broadly followed the protocol described by MARCUs et al. [21]. A coronal section was performed through the chest and from this "scout" sequence a horizontal long-axis view was obtained showing the four chambers of the heart. A series of short-axis images were then acquired perpendicular to the long-axis view, starting at the tricuspid valve and covering the complete right ventricle from base to apex. Short-axis image acquisition was triggered by the electrocardiogram $\mathrm{R}$ wave and performed in cine mode with a temporal resolution of $55 \mathrm{~ms}$, resulting in a series of images during the cardiac cycle at $55 \mathrm{~ms}$ intervals at each slice position. This was done by a segmented Flash with view-sharing to allow a reduced time resolution. Main parameters were repetition time (TR) 110, echo time (TE) 6.1, slice thickness $10 \mathrm{~mm}$, Flip Angle 20 degrees.

Fifteen consecutive heartbeats were required to acquire a set of images in each plane. Subjects were instructed to hold their breath during the acquisition of short-axis images. This resulted in a $12-15 \mathrm{~s}$ breathhold for image acquisition in each short-axis slice position and six and eight slices were required per patient. RVM and LVM measurements were thought unlikely to be affected by fluctuations in oxygen saturation, and therefore, because of logistical difficulties, MRI studies were performed without supplementary oxygen.

Magnetic resonance imaging analysis. RVM and LVM were calculated as follows [8]. The interventricular septum was taken to be part of the left ventricle. The cross-sectional area of right ventricular myocardium in each end-diastolic short-axis slice was calculated by manually tracing around the interior and exterior of the right ventricular free wall (fig. 1). The total volume of muscle for that slice was then obtained by multiplying the cross-sectional area by the slice thickness. This process was repeated for each shortaxis slice and the total volume of right ventricular myocardium calculated by summing all the slice volumes. RVM was then derived by multiplying the total volume by the specific density of cardiac muscle $\left(1.05 \mathrm{~g} \cdot \mathrm{cm}^{-3}\right)$. The moderator band was included in the calculation for RVM where it appeared. The process described above was repeated for the left ventricle to calculate LVM except that the cross-sectional area of myocardium in each slice was calculated by subtracting the area of the internal polygon from the external
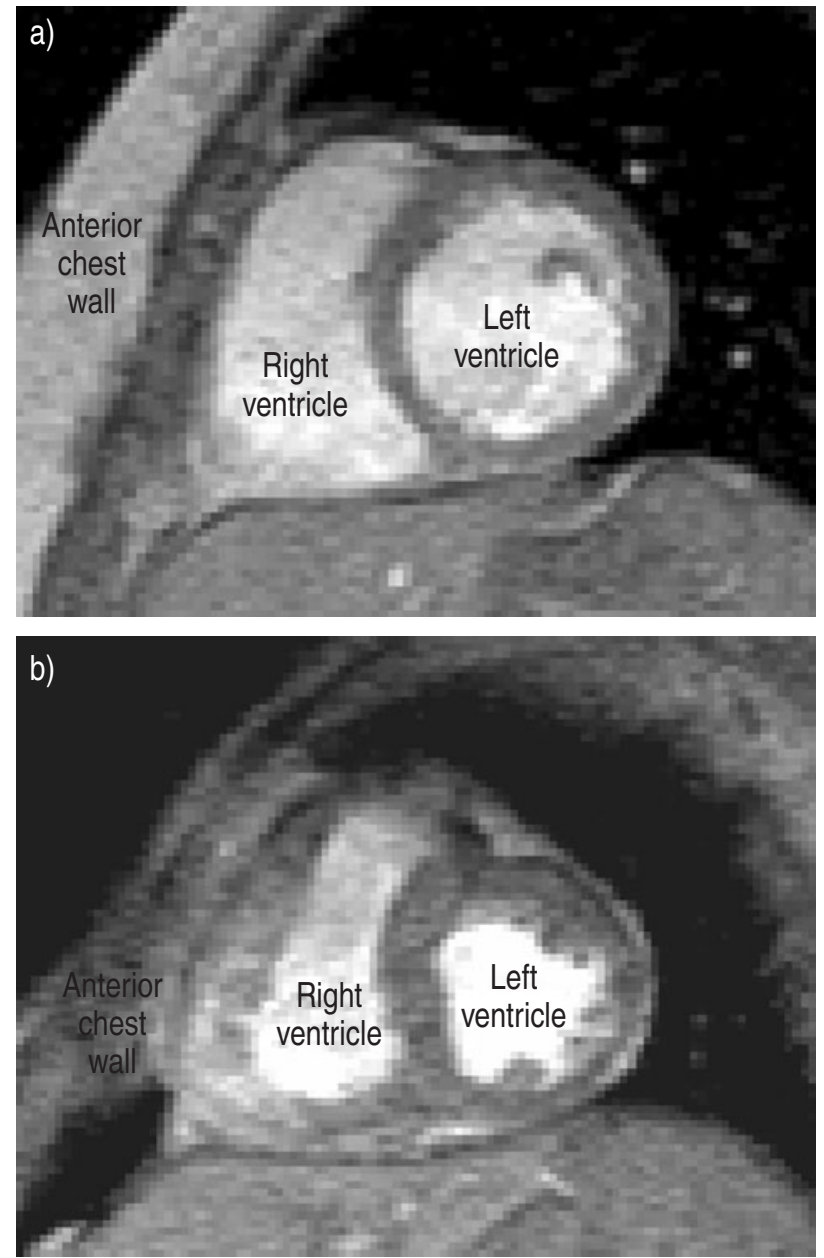

Fig. 1.-Examples of the end-diastolic short-axis images of the ventricles used to calculate right ventricular mass (RVM) and left ventricular mass (LVM) in a) a normal subject and b) a subject with pulmonary artery hypertension. The cross-sectional area of muscle in each slice was multiplied by slice thickness to obtain the slice muscle volume. This process was repeated for each slice and all the slice muscle volumes summed to obtain a total muscle volume for each ventricle and then multiplied by the known density of cardiac muscle $\left(1.05 \mathrm{~g} \cdot \mathrm{cm}^{-3}\right)$ to obtain ventricular mass.

(fig. 1). A VMI was then obtained by dividing RVM by LVM, a technique used in animal models of pulmonary hypertension [22] but not previously used in man.

\section{Statistical analysis}

Pearson's correlation coefficient and linear regression analysis were used to assess the relationship between MPAP measured at RHC and measurements made at ECHO and MRI (figs. 2-4, table 2). Subjects were also divided into those with normal MPAP at RHC (normals) and those with raised MPAP (PAHT). The two-sample unpaired t-test was used to assess the significance of differences between the two groups (table 3). An "intention-to-scan" analysis was carried out when calculating sensitivities and specificities (table 3). 


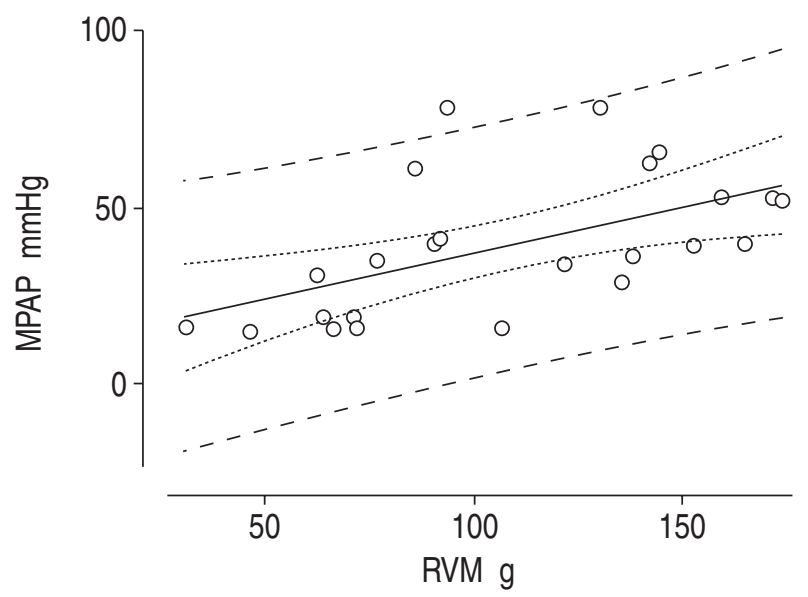

Fig. 2. - The relationship between mean pulmonary artery pressure (MPAP) and right ventricular mass (RVM). The Pearson correlation coefficient $(r)$ and linear regression analysis $(-)$ with mean confidence intervals $(\cdots \cdots \cdots \cdot)$ and individual prediction intervals $(--)$ are displayed. $\mathrm{r}=0.56 ; \mathrm{r}^{2}: 31.1 \%$.

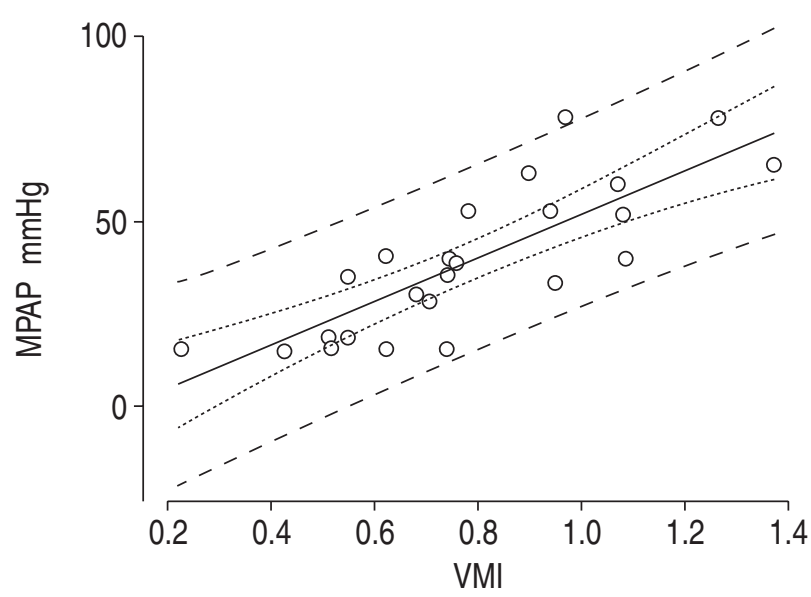

Fig. 3. - The relationship between mean pulmonary artery pressure (MPAP) and ventricular mass index (VMI). The Pearson correlation coefficient $(\mathrm{r})$ and linear regression analysis $(-)$ with mean confidence intervals $(. . . \ldots . . .$.$) and individual prediction intervals (- - -)$ are displayed. $r=0.81 ; r^{2}: 65.8 \%$.

\section{Results}

\section{General}

All twenty-six subjects underwent ECHO within 2 days of RHC. Twenty out of twenty-six subjects underwent MRI within 2 days of RHC. The remaining six were scanned up to 2 weeks later for logistical reasons (subjects $1,2,6,15,19$ and 21 ). Scanning was incomplete in one subject who was unable to breathhold adequately and has since died (subject 16), and was abandoned in another subject due to acute claustrophobia (subject 7).

\section{Ventricular mass}

The mean calculated values for RVM, LVM and VMI are shown in table 2 for 24 subjects. Measurements

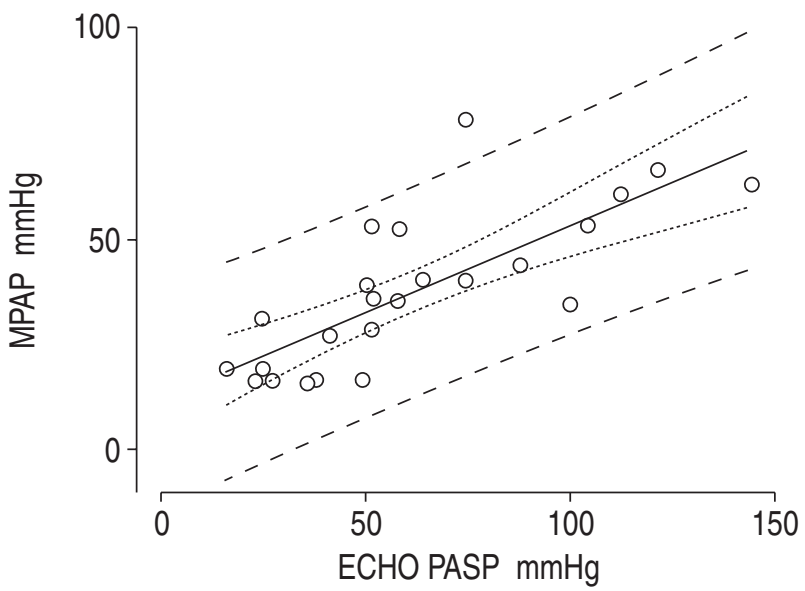

Fig. 4. - The relationship between mean pulmonary artery pressure (MPAP) and echocardiography pulmonary artery systolic pressure. The Pearson correlation coefficient (r) and linear regression analysis $(-)$ with mean confidence intervals $(. \cdots \ldots \ldots)$ and individual prediction intervals (- - ) are displayed. $\mathrm{r}=0.77 ; \mathrm{r}^{2}: 58.7 \%$.

were not possible in subject 16 due to difficulty breathholding and subject no. 7 due to claustrophobia. There was a significant difference between the two groups for RVM $(p<0.005)$ and VMI $(p<0.01)$, but not for LVM. There was a weak correlation between MPAP and RVM (r=0.56; fig. 2), but a much stronger correlation with reduced scatter between MPAP and VMI, ( $\mathrm{r}=0.81$; fig. 3). If a VMI of $>0.6$ is taken as abnormal there was one false negative result (subject 24) and two false positives (subjects 4 and 25) with two scanning failures (subjects 7 and 16), giving a sensitivity of $84 \%$ and a specificity of $71 \%$ (table 3 ).

\section{Doppler echocardiography}

The values for estimated PASP are shown in table 1 and statistical analysis in tables 2 and 3 . Values are listed for 24 subjects; subject 6 was reported as $<25$ and a value of 25 was used for statistical analysis. In subject 8, estimated PASP was raised but could not be accurately measured, and was therefore not included in linear regression analysis. PASP could not be measured in subject 22 . There was a significant difference between the two groups $(\mathrm{p}<0.0001)$ and a good correlation with MPAP as shown in figure $4(\mathrm{r}=0.77)$ and table 3 . However there were three false positive

Table 2.-Correlations with invasive mean pulmonary artery pressure (MPAP) and sensitivity and specificity for pulmonary arterial hypertension (PAHT)

\begin{tabular}{lcccc}
\hline & $\begin{array}{c}\text { Subjects } \\
\text { n }\end{array}$ & $\begin{array}{c}\text { Correlation } \\
\text { with } \\
\text { MPAP }\end{array}$ & $\begin{array}{c}\text { Sensitivity } \\
\text { for } \\
\text { PAHT \% }\end{array}$ & $\begin{array}{c}\text { Specificity } \\
\text { for } \\
\text { PAHT \% }\end{array}$ \\
\hline VMI & 26 & 0.81 & 84 & 71 \\
$\begin{array}{c}\text { ECHO PASP } \\
\text { mmHg }\end{array}$ & 26 & 0.77 & 89 & 57 \\
\hline
\end{tabular}

VMI: ventricular mass index; ECHO: echocardiography; PASP: pulmonary artery systolic pressure. 
Table 3. - Comparison of normals versus pulmonary arterial hypertension (PAHT) patients

\begin{tabular}{|c|c|c|c|c|c|}
\hline & \multicolumn{2}{|c|}{ Normals } & \multicolumn{2}{|c|}{ PAHT } & \multirow[t]{2}{*}{$\mathrm{p}$-value } \\
\hline & Mean \pm SD & Range & Mean \pm SD & Range & \\
\hline Subjects $n$ & 7 & 7 & 19 & 19 & \\
\hline Age yrs & $50 \pm 11$ & $32-64$ & $50 \pm 12$ & $29-68$ & NS \\
\hline MPAP $\mathrm{mmHg}$ & $17 \pm 2$ & $15-19$ & $47 \pm 16$ & $27-78$ & $<0.0001$ \\
\hline $\mathrm{CO} \mathrm{L} \cdot \mathrm{min}^{-1}$ & $5.1 \pm 1.2$ & $3.4-6.8$ & $4.4 \pm 1.3$ & $2.6-6.7$ & NS \\
\hline RVM g & $66 \pm 24$ & $31-107$ & $126 \pm 36$ & $63-174$ & $<0.005$ \\
\hline LVM g & $129 \pm 24$ & $98-172$ & $145 \pm 41$ & $81-205$ & NS \\
\hline VMI & $0.5 \pm 0.2$ & $0.2-0.7$ & $0.9 \pm 0.2$ & $0.6-1.4$ & $<0.01$ \\
\hline ECHO PASP mmHg & $31 \pm 11$ & $16-49$ & $75 \pm 33$ & $25-144$ & $<0.0001$ \\
\hline
\end{tabular}

MPAP: mean pulmonary artery pressure; CO: cardiac output; RVM: right ventricular mass; LVM: left ventricular mass; VMI: ventricular mass index; ECHO: echocardiography; PASP: pulmonary artery systolic pressure.

results (subjects 4, 11 and 25), one failed scan (subject 22) and one false negative result (subject 13), giving an overall sensitivity of $89 \%$ and a specificity of $57 \%$ (table 3).

\section{Discussion}

Calculated VMI measured with MRI is a new clinical tool that provides an accurate and practical means of estimating MPAP noninvasively in subjects with primary and secondary pulmonary arterial hypertension with a wide range of pressures and diagnoses. VMI correlated well with MPAP at right heart catheterisation and although confidence and prediction intervals are relatively wide indicating limited precision, they are narrower than for Doppler ECHO in the same group of subjects. The method used for indexing RVM has not been previously described in pulmonary hypertension. A closer agreement for the VMI was found in the present study when compared to that reported by KATZ et al. [8] who used a RVM index calculated by dividing RVM by body surface area in a study of eight adults and five children with PPH. This may be because pulmonary arterial hypertension also has an effect on the morphology and function of the left ventricle. The study has also shown that the VMI may be more specific for pulmonary arterial hypertension than Doppler ECHO, the current gold standard noninvasive investigation. VMI was also more sensitive than Doppler ECHO in those patients in whom scanning was successfully completed (94 versus $89 \%$ excluding subjects 7 and 16).

This is the first study to suggest a practical advantage of MRI over Doppler ECHO in the routine assessment of pulmonary haemodynamics. The reason for this may be that VMI reflects the right ventricular response to a given pulmonary artery pressure sustained over a long period. It is therefore unlikely to be influenced by short-term physiological variables such as cardiac frequency, posture, hydration status or supplementary oxygen, all of which may affect the real-time pressure estimates made by Doppler ECHO. Although Doppler ECHO is a relatively cheap and practical method of assessing acute changes in pulmonary haemodynamics, MRI is more likely to provide a reliable assessment of long-term disease progression and response to treatment because ventricular mass will not respond to transient changes in pulmonary artery pressure.

MRI does have limitations but was well tolerated by almost all the subjects. The problems of noise, claustrophobia and prolonged breath-holding are fast diminishing with advancing technology. The presence of unsecured ferromagnetic material in the body remains a contraindication. Many patients with pulmonary hypertension are treated with continuous intravenous or subcutaneous prostanoids requiring a syringe pump, which would have to remain outside the scanning room.

There are several possible sources of error in this study. No attempt was made to blind the reporting of scans which may have led to bias and the interobserver reproducibility was not tested. Some difficulty was encountered in delineating the right ventricular lumen due to the presence of papillary muscles, in particular at the apex of the ventricle, but this was minimised by adjusting the contrast appropriately. This may have led to a tendency to overestimate RVM. All the values for LVM fell within the normal ranges suggested recently by LORENZ et al. [5] however only three of the seven subjects without pulmonary hypertension at rest fell within the suggested normal range for right RVM. This may suggest overestimation of RVM in the study, however, a similar range of values to those reported by KATZ et al. [8] were found. It should also be remembered that all the "normal" subjects were initially referred with clinical evidence of pulmonary vascular disease. Since short-axis sections for both ventricles were taken perpendicular to the long axis of the left ventricle, this could have led to oblique sections being taken through the right ventricle and inaccuracies in slice volume estimation. Seven of the subjects had ischaemic heart disease or systemic hypertension, possibly altering LVM and consequently VMI. However there was no evidence of left ventricular dysfunction or hypertrophy at ECHO and right heart catheterisation. Although subjects 16, 17, 20 and 21 only received supplementary oxygen during Doppler ECHO and RHC, and not during MRI, this is unlikely to have affected the anatomical measurements. 
It has been shown that magnetic resonance imaging may be more accurate than Doppler echocardiography, with similar or better sensitivity and specificity for both primary and secondary forms of the disease. Although Doppler echocardiography was successfully performed in almost all subjects in this study, a failure rate as high as $60 \%$ is known to exist in some patient groups due to body habitus, the absence of a tricuspid regurgitant jet or coexisting lung disease [3]. These are not limiting factors for MRI which may provide a useful alternative for monitoring patients and screening high-risk individuals in whom Doppler echocardiography is unhelpful. Indeed, with wider availability, reduced costs and the advent of modern scanners it could become the method of choice.

\section{References}

1. D'Alonzo GE, Barst RJ, Ayres SM, et al. Survival in patients with primary pulmonary hypertension. Results from a national prospective registry. Ann Intern Med 1991; 115: 343-349.

2. Yock PG, Popp RL. Noninvasive estimation of right ventricular systolic pressure by Doppler ultrasound in patients with tricuspid regurgitation. Circulation 1984; 70: 657-662.

3. Torbicki A, Skwarski K, Hawrylkiewicz I, Pasierski T, Miskiewicz Z, Zielinski J. Attempts at measuring pulmonary arterial pressure by means of Doppler echocardiography in patients with chronic lung disease. Eur Respir J 1989; 2: 856-860.

4. Raeside DA, Chalmers G, Clelland J, Madhok R, Peacock AJ. Pulmonary artery pressure variation in patients with connective tissue disease: 24 hour ambulatory pulmonary artery pressure monitoring. Thorax 1998; 53: 857-862.

5. Lorenz $\mathrm{CH}$, Walker ES, Morgan VL, Klein SS, Graham TP Jr. Normal human right and left ventricular mass, systolic function, and gender differences by cine magnetic resonance imaging. $J$ Cardiovasc Mag Res 1999; 1: 7-21.

6. Boxt LM, Katz J, Kolb T, Czegledy FP, Barst RJ. Direct quantitation of right and left ventricular volumes with nuclear magnetic resonance imaging in patients with primary pulmonary hypertension. $J \mathrm{Am}$ Coll Cardiol 1992; 19: 1508-1515.

7. Boxt LM, Katz J. Magnetic resonance imaging for quantitation of right ventricular volume in patients with pulmonary hypertension. J Thorac Imaging 1993; 8: 92-97.

8. Katz J, Whang J, Boxt LM, Barst RJ. Estimation of right ventricular mass in normal subjects and in patients with primary pulmonary hypertension by nuclear magnetic resonance imaging. $\mathrm{J} \mathrm{Am}$ College Cardiol 1993; 21: 1475-1481.

9. Kondo C, Caputo GR, Masui T, et al. Pulmonary hypertension: pulmonary flow quantification and flow profile analysis with velocity-encoded cine MR imaging. Radiology 1992; 183: 751-758.

10. Tardivon AA, Mousseaux E, Brenot F, et al. Quantification of hemodynamics in primary pulmonary hypertension with magnetic resonance imaging. $\mathrm{Am}$ J Respir Crit Care Med 1994; 150: 1075-1080.

11. Paz R, Mohiaddin RH, Longmore DB. Magnetic resonance assessment of the pulmonary arterial trunk anatomy, flow, pulsatility and distensibility. Eur Heart $J$ 1993; 14: 1524-1530.

12. Moulton MJ, Creswell LL, Ungacta FF, Downing SW, Szabo BA, Pasque MK. Magnetic resonance imaging provides evidence for remodeling of the right ventricle after single-lung transplantation for pulmonary hypertension. Circulation 1996; 94: II312-II319.

13. Frist WH, Lorenz $\mathrm{CH}$, Walker ES, et al. MRI complements standard assessment of right ventricular function after lung transplantation. Ann Thorac Surg 1995; 60: 268-271.

14. Mohiaddin RH, Paz R, Theodoropoulos S, Firmin $\mathrm{DN}$, Longmore DB, Yacoub MH. Magnetic resonance characterization of pulmonary arterial blood flow after single lung transplantation. $J$ Thorac Cardiovasc Surg 1991; 101: 1016-1023.

15. Mohiaddin RH. Magnetic resonance imaging of single lung transplantation. Rays 1994; 19: 405-414.

16. Bellenger NG, Marcus NJ, Davies C, Yacoub M, Banner NR, Pennell DJ. Left ventricular function and mass after orthotopic heart transplantation: a comparison of cardiovascular magnetic resonance with echocardiography. J Heart Lung Transplant 2000; 19: $444-452$.

17. Frank H, Globits S, Glogar D, Neuhold A, Kneussl M, Mlczoch J. Detection and quantification of pulmonary artery hypertension with MR imaging: results in 23 patients. AJR 1993; 161: 27-31.

18. Bouchard A, Higgins CB, Byrd BFD, Amparo EG, Osaki L, Axelrod R. Magnetic resonance imaging in pulmonary arterial hypertension. Am J Cardiol 1985; 56: 938-942.

19. Saito H, Dambara T, Aiba M, Suzuki T, Kira S. Evaluation of cor pulmonale on a modified short-axis section of the heart by magnetic resonance imaging. Am Rev Respir Dis 1992; 146: 1576-1581.

20. Murray TI, Boxt LM, Katz J, Reagan K, Barst RJ. Estimation of pulmonary artery pressure in patients with primary pulmonary hypertension by quantitative analysis of magnetic resonance images. $J$ Thorac Imaging 1994; 9: 198-204.

21. Marcus JT, Vonk Noordegraaf A, De Vries PM, et al. MRI evaluation of right ventricular pressure overload in chronic obstructive pulmonary disease. J Magn Reson Imaging 1998; 8: 999-1005.

22. Wanstall JC, O'Donnell SR, Kay CS. Increased relaxation by felodipine on pulmonary artery from rats with monocrotaline-induced pulmonary hypertension does not reflect functional impairment of the endothelium. Pulm Pharmacol 1991; 4: 60-66. 